\title{
Stage II Pancreatic Cancer: Radical, Palliative Surgery or Stenting?
}

\author{
Audrius Šileikis' ${ }^{1}$ Benediktas Kurlinkus², Marius Kryžauskas' ${ }^{1}$, Kęstutis Strupas ${ }^{1}$ \\ ${ }^{1}$ Vilnius University Center of Abdominal Surgery, Vilnius, Lithuania \\ ${ }^{2}$ Vilnius University Center of Gastroenterology and Hepatology, Vilnius, Lithuania \\ Email: Audrius.Sileikis@santa.It, Benediktas.Kurlinkus@santa.It
}

Received 30 November 2015; accepted 26 December 2015; published 29 December 2015

Copyright (C) 2015 by authors and Scientific Research Publishing Inc.

This work is licensed under the Creative Commons Attribution International License (CC BY). http://creativecommons.org/licenses/by/4.0/

(c) (i) Open Access

\section{Abstract}

Background: Pancreatic ductal adenocarcinoma is the fourth most common reason of death among oncological diseases with ever increasing mortality. At the time of diagnosis, patients are usually suitable for three ways of treatment: radical, palliative surgery or stenting. Deciding the best option depends on clinical situation, but is still a matter of debate. Methods: We performed a retrospective research of patients with stage II pancreatic head cancer treated in our clinic between years 2002-2014. Four groups were formed according to the used treatment method: group A: radical surgery with R0 (microscopic tumour clearance) margin; group B: radical surgery with R1 (presence of tumour cells within $1 \mathrm{~mm}$ of the resection margin) margin; group C: biliary tract stenting; group D: biliodigestive anastomosis. Clinical data and most importantly the survival of these patients were compared. Results: 200 patients were involved in the final analysis, 82 (41\%) of them were IIA and 118 (59\%) were IIB. Group A consisted of 113 patients; group B consisted of 28 patients; group C consisted of 33 patients; group D consisted of 26 patients. In patients with IIA stage, group $A$ had the highest survival rate compared with other groups, mean survival was 3.242 versus 1.600; 0.454; 0.652 years. Patients with IIB stage of cancer similarly had longer survival in group A versus other groups, 1.720 versus $0.931 ; 0.713 ; 0.957$ years. Conclusions: Patients with IIA and IIB stage of pancreatic cancer benefit the most from radical surgery with R0 margin. However, for patients with lymph node involvement (stage IIB) and when achieving Ro margin is hardly possible, neoadjuvant treatment seems promising, but we need further randomized controlled trials to fully confirm its effectiveness.

\section{Keywords}

Pancreatic Ductal Adenocarcinoma, Radical Surgery, Palliative Surgery, Biliary Tract Stenting 


\section{Introduction}

There is a worldwide tendency to increasing morbidity because of the malignant pancreatic tumours [1] [2]. Pancreatic ductal adenocarcinoma (PDA) is the most frequent type in this group [3]. Morbidity of PDA is not as high as the morbidity of other malignant tumours (13 yearly cases per 100,000 persons) [4]. Despite that, this disease is the fourth most common reason of death among oncological diseases (it has high morbidity/mortality ratio) [4]. Radical surgery remains a golden standard for its treatment. Unfortunately, it is only possible to perform it for $15 \%-20 \%$ of patients [5]. The prognosis is poor even for patients with early diagnosed disease: 5 years survival is $25 \%$ - $30 \%$ without metastasis in the regional lymph nodes and $10 \%$ with metastasis in the regional lymph nodes [5]. The remaining patients, unsuitable for radical surgery, have two ways of palliative treatment: biliary tract stenting or biliodigestive anastomosis [6] [7]. Deciding the best option depends on tumour stage, patient preferences, age, clinical situation and the estimated prognosis, but is still a matter of debate. This highlights the importance of survival analysis done so far comparing patients undergoing these different treatment methods [8]-[10]. However, there are still no clear indications when which method should be used.

For this reason the objective of this paper is to determine which patients with a certain stage of pancreatic cancer can benefit the most in terms of survival from a particular treatment method.

\section{Methods}

A retrospective research was accomplished in the Vilnius University Hospital Santariskiu Clinics. It was approved by the local bioethics committee. Medical data of patients who underwent radical, palliative surgery or stenting of pancreatic head cancer between years 2002-2014 was collected. The pathology reports for all these patients were reviewed and only those patients with a histologically approved diagnosis of pancreatic ductal adenocarcinoma of the head of pancreas were selected for inclusion within this study. Patients for whom surgery or stenting was performed because of duodenal or ampullary tumour, cholangiocarcinoma, pancreas neuroendocrine tumour or any other pancreatic pathology were excluded from the study. Also patients with intraoperative v. mesenterica superior and/or v. portae resections were excluded. Four groups were formed according to the used method of treatment: group A-pancreatoduodenal resection (PDR) with R0 margin; group B-PDR with R1 margin; group C-biliary tract stenting, group D-biliodigestive anastomosis. All patients, undergoing PDR, had an urgent pathological examination, which includes sending specimens from resection margins of pancreatic stump, mesoduodenum at the level of v. mesenterica superior and common bile duct to the pathology center for a quick analysis. From the data of patients included in the final analysis these variables were selected: size of primary tumour (pT1-3) and metastasis in the regional lymph nodes (pN0-1) which were pathologically proved for patients who underwent PDR or by computed tomography (CT) scan determined for those with palliative treatment. Tumour resection margins (R0-1) were investigated only for patients who undergone PDR. According to this information we defined the stage of the tumour (using TNM 10 classification of malignant tumours). None of the patients received neoadjuvant chemotherapy. After surgery patients underwent adjuvant treatment. Patients were followed for survival, data were received from The Residents Register Service under the Ministry of the Interior of the Republic of Lithuania. Uni-variate Kaplan-Meier analysis were performed and Kaplan-Meier survival curves were drawn for both groups of patients with IIA and IIB stages of cancer. Statistical significance was determined using the Log-rank (Mantel-Cox) test. Statistical significance was set at a level of p $<0.05$. Data analyses were performed using Microsoft Excel 2013 and SPSS 17.0.

\section{Results}

Between years 2002 and 2014200 patients with stage II pancreatic ductal adenocarcinoma were treated in our clinic. 82 (41\%) of them were IIA and 118 (59\%) were IIB. Four groups were formed: group A with 113 patients; groups B with 28 patients; group C with 33 patients; group D with 26 patients. The four groups were similar with respect to sex and tumour size (Table 1). There were statistically significant distinction in age between groups with patients undergoing PDR and palliative treatment methods (Table 1). This is probably due to our clinic practice to perform PDR under the age of 75 years. Two of these younger patients refused PDR and choose biliodigestive anastomosis and stenting of biliary tract instead. Older patients usually are recommended to undergo palliative treatment. Preferences for recommended palliative treatment methods varied in our clinic due to changing international opinion about the most effective method to palliate throughout time [11] [12]. 
Table 1. Comparison of patients groups.

\begin{tabular}{ccccc}
\hline & $\begin{array}{c}\text { Group A } \\
\text { (PDR with R0 margin) }\end{array}$ & $\begin{array}{c}\text { Group B } \\
\text { (PDR with R1 margin) }\end{array}$ & $\begin{array}{c}\text { Group C } \\
\text { (biliary tract stenting) }\end{array}$ & $\begin{array}{c}\text { Group D } \\
\text { (biliodigestive anastomosis) }\end{array}$ \\
\hline $\begin{array}{c}\text { Number } \\
\text { Age (years) }\end{array}$ & 113 & 28 & 33 & 26 \\
Sex & $64.67 \pm 9$ & $64.34 \pm 9$ & $76 \pm 10^{\mathrm{a}}$ & $75 \pm 10^{\mathrm{a}}$ \\
Male & $64(57 \%)$ & & & $9(35 \%)$ \\
Female & $49(43 \%)$ & $17(61 \%)$ & $17(52 \%)$ & $17(65 \%)$ \\
Stage & & $11(39 \%)$ & $16(48 \%)$ & $12(46 \%)$ \\
IIA & $37(33 \%)$ & $12(43 \%)$ & $21(64 \%)$ & $14(54 \%)$ \\
IIB & $76(67 \%)$ & $16(57 \%)$ & $12(36 \%)$ & $0(0 \%)$ \\
T & $1(1 \%)$ & & & $0(0 \%)$ \\
1 & $3(3 \%)$ & $0(0 \%)$ & $2(0 \%)$ & $26(100 \%)$ \\
\hline
\end{tabular}

${ }^{\mathrm{a}} \mathrm{p}<0.05$ vs. PDR methods.

From 141 pancreatoduodenal resections performed in our clinic for the stage II pancreatic cancer 8 (20\%) had microscopically positive resection margin in the final pathological examination. In our pathological examination protocol it is defined as a distance of the tumour from the resection margin of $\leq 1 \mathrm{~mm}$.

In IIA stage patients, group A had the highest survival rate compared with other groups, mean survival 3.242 versus 1.600; 0.454; 0.652 years (Table 2, Figure 1(a)). It reached statistical significance with p value $<0.0001$. Patients with IIB stage of cancer similarly had longer survival in group A versus other groups, 1.720 vs. 0.931; 0.713; 0.957 years (Table 2, Figure 1 (b)). It also reached statistical significance with p value of 0.002 . Survival of group A and group B for 3 and 5 years is shown in Table 3, survival of group C and group D for 1 year is shown in Table 4.

\section{Discussion}

With the growing incidence of obesity and diabetes increases the morbidity of pancreatic cancer. For this reason well-timed diagnosis and proper methods of treatment remains an issue. Currently the main way of treatment able to change its natural course is PDR. Unfortunately, this operation is associated with high mortality and frequent complications [13]-[15]. Consequently, we decided to analyse our data and determine if it is reasonable to perform PDR for all potentially resectable patients.

Our analysis showed that R0 resection is the best way of treatment for patients with both IIA and IIB stages of pancreatic cancer (survival in IIA stage was 3.242 years and 1.720 years in IIB stage). Unfortunately, to achieve R0 resection while performing PDR is not always feasible, despite the intraoperative urgent pathological examination of resection margins. In our study $20 \%$ of patients were R1. Information in the present literature about the frequency of microscopically positive resection margin while performing pancreaticoduodenectomy for pancreatic cancer is very different: from $19 \%$ [16] to $82 \%$ [17]. As Pang et al. identified, intra-operative frozen section increases the overall R0 rate but usually does not improve survival. This might be because in some cases when re-excision couldn't be performed easily and safely patients had negative pancreatic neck margins after re-excision, but this did not guarantee that there is no other involved margins, for example from mesoduodenum [18]. Supposedly, it is a result of precise pathological examination [19]-[21]. However, recently Merkow et al. evaluated 1002 hospitals and 14889 patients who underwent radical surgery for PDA and concluded that despite differences in pathologic examination techniques, hospitals can reliably provide comparative data on surgical margin status [22]. Therefore, in some cases, R1 resection is imminent and unavoidable. Especially then cancer is at the boundary of pancreatic head and neck while spreading to the mesoduodenum.

From our experience, for stage IIA pancreatic cancer even PDR with R1 resection margin is more acceptable in terms of survival than palliative treatment. For those patients with stage IIB, when achieving R0 margin at PDR is hardly possible, biliodigestive anastomosis together with neoadjuvant chemotherapy could be suggested 
Table 2. Survival of patients groups with IIA and IIB stage pancreatic cancer.

\begin{tabular}{ccccc}
\hline & Group A & Group B & Group C & \\
\hline IIA stage & & & \\
Number & 37 & 12 & 12 & $0.454 \pm 0.1$ \\
Mean survival (years) & $3.242 \pm 0.6$ & $1.600 \pm 0.4$ & 12 \\
IIB stage & 76 & 16 & 0.2 \\
Number & $1.720 \pm 0.2$ & $0.931 \pm 0.1$ & $0.713 \pm 0.1$ \\
Mean survival (years) & & & $0.957 \pm 0.2$ \\
\hline
\end{tabular}

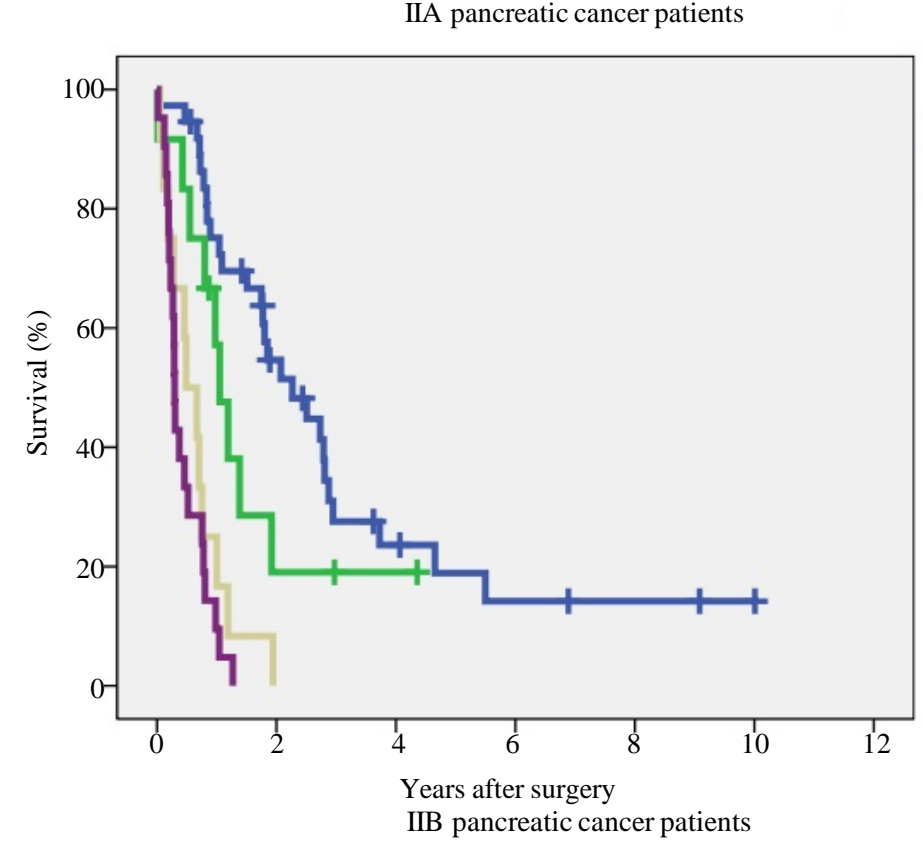

(a)

\section{$\boldsymbol{n P D R}$ with R0}

$\rightarrow$ PDR with R1

Biliodigestive anastomosis

Stenting

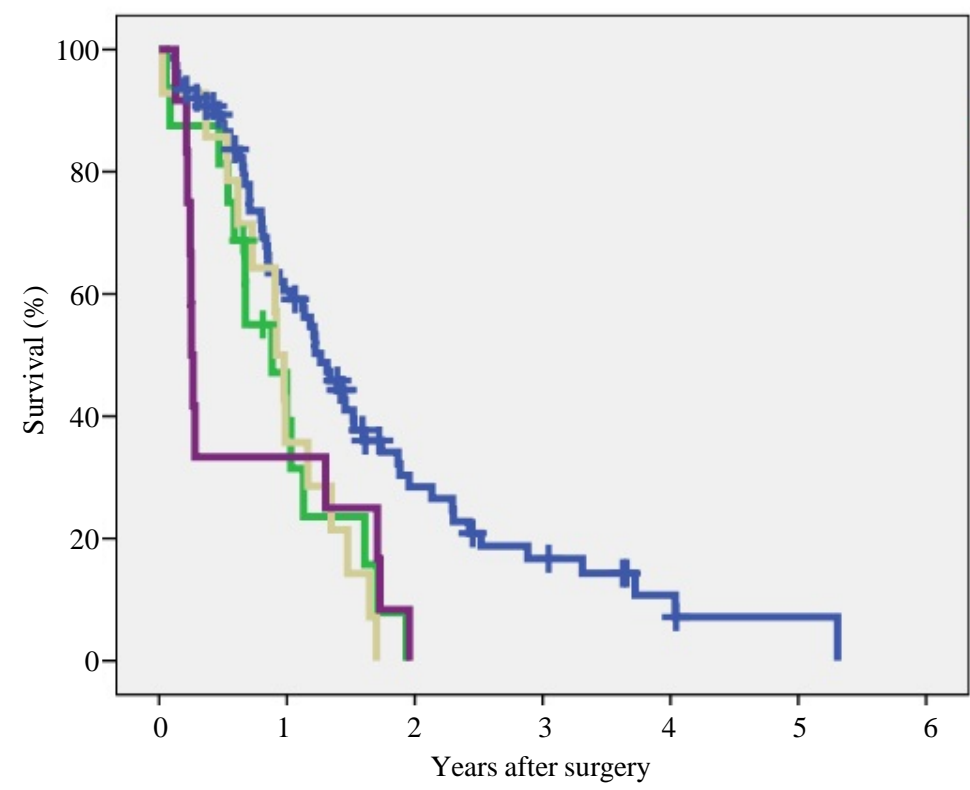

(b)

-PDR with R0

$=$ PDR with R1

Biliodigestive

anastomosis

7 Stenting

Figure 1. (a) Kaplan-Meier survival curves of IIA pancreatic cancer patients; (b) Kaplan-Meier survival curves of IIB pancreatic cancer patients. 
Table 3. Survival of patients groups A and B for 3 and 5 years.

\begin{tabular}{ccc}
\hline & Group A & Group B \\
\hline IIA stage & & \\
3 years & $21.62 \%$ & $16.60 \%$ \\
5 years & $10.81 \%$ & $0 \%$ \\
IIB stage & & \\
3 years & $10.53 \%$ & $0 \%$ \\
5 years & $1.32 \%$ & $0 \%$ \\
\hline
\end{tabular}

Table 4. Survival of patients groups C and D for 1 year.

\begin{tabular}{ccc}
\hline & Group C & Group D \\
\hline IIA stage & & \\
1 years & $22.23 \%$ & $36.77 \%$ \\
IIB stage & & $18.31 \%$ \\
1 years & $12.81 \%$ & \\
\hline
\end{tabular}

as an alternative (survival of biliodigestive anastomosis group $0.957 \pm 0.2$ years vs. $0.931 \pm 0.1$ years survival in PDR with R1 margin group). Accordingly, it seems that for some patients with IIB stage neoadjuvant chemotherapy is advisable. However, the randomized controlled trials done so far is not comprehensive enough to prove its effectiveness [23]-[25]. Moreover, for some stented patients it is difficult to accurately determine lymph nodes involvement in CT scans due to cholangitis. Hence, deciding which is better in stage IIB pancreatic cancer, PDR with R1 resection margin or biliodigestive anastomosis together with neoadjuvant chemotherapy is still a matter of debate. Supposedly, results of neoadjuvant chemotherapy will improve with introduction of new chemotherapy agents.

Other important issue is the question which alternative palliative treatment method should be suggested for patients refusing to undergo PDR or when because of comorbidity it is contraindicated. Palliation in this respect remains the main line of treatment. An alternative to palliative surgery alone have emerged for the relieving of jaundice and cholangitis. This is the introduction of nonoperative treatment method for decompressing the obstructed biliary tree such as stenting. Which is the most efficient type of palliation remains controversial in terms of long-term patient benefit, especially in cases when un-resectability is determined during surgery. Smith et al. determined that patients with biliary tract stenting had a significantly decreased complication rate (11\% vs. 29\%) and lower with a procedure related mortality (3\% vs. 14\%) [11]. On the other hand, a meta-analysis of three studies [11] [26] [27] done by Taylor et al. compared endoscopically performed stenting with surgical biliodigestive anastomosis for malignant biliary tract obstruction and discovered a reintervention rate of 3\% in surgically treated patients vs. $36 \%$ in stented patients [12]. This shows more than tenfold increased risk of additional interventions required after endoscopic stenting. It also represents our analysis data, which shows higher survival in patients group with biliodigestive anastomosis, both with IIA and IIB stage pancreatic cancer (0.652 \pm 0.2 vs. $0.454 \pm 0.1$ years in IIA stage and $0.957 \pm 0.2$ vs. $0.713 \pm 0.1$ years in IIB stage)

We can point out factors which could affect the survival of the patients. Firstly, it is improved intraoperative urgent pathological examination to standardize it more between different pathology centers. Secondly, seeking for R0 resection margin by improving surgical technique-surgeon must perform at least 10 PDR annually [28]. Finally, it is personal medicine-targeted neoadjuvant or adjuvant chemotherapy according to gene mutations [29]-[31].

We understand the limitation of the paper. It is the retrospective analysis of data, mean age difference between groups with patients undergoing PDR and palliative treatment methods, no analysis of perioperative and postoperative complications and no data about patients life quality after treatment.

\section{Conclusion}

Patients with IIA and IIB stage of pancreatic cancer benefit the most from radical surgery with R0 margin. 
However, for patients with lymph node involvement (stage IIB) and when achieving R0 margin is hardly possible, neoadjuvant treatment seems promising, but we need further randomized controlled trials to fully confirm its effectiveness.

\section{References}

[1] Bosetti, C., Bertuccio, P., Malvezzi, M., Levi, F., Chatenoud, L., et al. (2013) Cancer Mortality in Europe, 2005-2009, and an Overview of Trends since 1980. Annals of Oncology, 24, 2657-2671. http://dx.doi.org/10.1093/annonc/mdt301

[2] Raimondi, S., Maisonneuve, P. and Lowenfels, A.B. (2009) Epidemiology of Pancreatic Cancer: An Overview. Nature reviews. Gastroenterology \& Hepatology, 6, 699-708. http://dx.doi.org/10.1038/nrgastro.2009.177

[3] Sohn, T.A., Yeo, C.J., Cameron, J.L., Koniaris, L., Kaushal, S., et al. (2000) Resected Adenocarcinoma of the Pancreas-616 Patients: Results, Outcomes, and Prognostic Indicators. Journal of Gastrointestinal Surgery, 4, 567-579. http://dx.doi.org/10.1016/S1091-255X(00)80105-5

[4] Yadav, D. and Lowenfels, A.B. (2013) The Epidemiology of Pancreatitis and Pancreatic Cancer. Gastroenterology, 144, 1252-1261. http://dx.doi.org/10.1053/j.gastro.2013.01.068

[5] Zuckerman, D.S. and Ryan, D.P. (2008) Adjuvant Therapy for Pancreatic Cancer: A Review. Cancer, 112, $243-249$. http://dx.doi.org/10.1002/cncr.23174

[6] Assfalg, V., Hüser, N., Michalski, C., Gillen, S., Kleeff, J., et al. (2011) Palliative Interventional and Surgical Therapy for Unresectable Pancreatic Cancer. Cancers, 3, 652-661. http://dx.doi.org/10.3390/cancers3010652

[7] Hüser, N., Michalski, C.W., Schuster, T., Friess, H. and Kleeff, J. (2009) Systematic Review and Meta-Analysis of Prophylactic Gastroenterostomy for Unresectable Advanced Pancreatic Cancer. British Journal of Surgery, 96, 711719. http://dx.doi.org/10.1002/bjs.6629

[8] Tol, J.A.M.G, Eshuis, W.J., Besselink, M.G.H., van Gulik, T.M., Busch, O.R.C., et al. (2015) Non-Radical Resection versus Bypass Procedure for Pancreatic Cancer-A Consecutive Series and Systematic Review. European Journal of Surgical Oncology, 41, 220-227. http://dx.doi.org/10.1016/j.ejso.2014.11.041

[9] Nordby, T., Ikdahl, T., BowitzLothe, I.M., Fagerland, M.W., Heiberg, T., et al. (2013) Improved Survival and Quality of Life in Patients Undergoing R1 Pancreatic Resection Compared to Patients with Locally Advanced Unresectable Pancreatic Adenocarcinoma. Pancreatology, 13, 180-185. http://dx.doi.org/10.1016/j.pan.2013.01.003

[10] Wang, S., Shyr, Y., Su, C., Chen, T. and Wu, C. (2012) Palliative Pancreaticoduodenectomy in Pancreatic and Periampullary Adenocarcinomas. Pancreas, 41, 882-887. http://dx.doi.org/10.1097/MPA.0b013e31823c9d46

[11] Smith, A.C., Dowsett, J.F., Russell, R.C., Hatfield, A.R. and Cotton, P.B. (1994) Randomised Trial of Endoscopic Stenting versus Surgical Bypass in Malignant Low Bileduct Obstruction. The Lancet, 344, 1655-1660. http://dx.doi.org/10.1016/S0140-6736(94)90455-3

[12] Taylor, M.C., McLeod, R.S. and Langer, B. (2000) Biliary Stenting versus Bypass Surgery for the Palliation of Malignant Distal Bile Duct Obstruction: A Meta-Analysis. Liver Transplantation, 6, 302-308. http://dx.doi.org/10.1053/lv.2000.5196

[13] Ball, C.G., Dixon, E., Vollmer, C.M. and Howard, T.J. (2015) The View from 10,000 Procedures: Technical Tips and Wisdom from Master Pancreatic Surgeons to Avoid Hemorrhage during Pancreaticoduodenectomy. BMC Surgery, 15, 122. http://dx.doi.org/10.1186/s12893-015-0109-y

[14] Kantor, O., Talamonti, M.S., Stocker, S.J., Wang, C., Winchester, D.J., et al. (2015) A Graded Evaluation of Outcomes Following Pancreaticoduodenectomy with Major Vascular Resection in Pancreatic Cancer. Journal of Gastrointestinal Surgery, in press. http://dx.doi.org/10.1007/s11605-015-2957-2

[15] Okano, K., Hirao, T., Unno, M., Fujii, T., Yoshitomi, H., et al. (2015) Postoperative Infectious Complications after Pancreatic Resection. British Journal of Surgery, 102, 1551-1560. http://dx.doi.org/10.1002/bjs.9919

[16] Neoptolemos, J.P., Stocken, D.D., Dunn, J.A., Almond, J., Beger, H.G., et al. (2001) Influence of Resection Margins on Survival for Patients with Pancreatic Cancer Treated by Adjuvant Chemoradiation and/or Chemotherapy in the ESPAC-1 Randomized Controlled Trial. Annals of Surgery, 234, 758-768. http://dx.doi.org/10.1097/00000658-200112000-00007

[17] Jamieson, N.B., Denley, S.M., Logue, J., MacKenzie, D.J., Foulis, A.K., et al. (2011) A Prospective Comparison of the Prognostic Value of Tumor- and Patient-Related Factors in Patients Undergoing Potentially Curative Surgery for Pancreatic Ductal Adenocarcinoma. Annals of Surgical Oncology, 18, 2318-2328. http://dx.doi.org/10.1245/s10434-011-1560-3

[18] Pang, T.C.Y., Wilson, O., Argueta, M.A., Hugh, T.J., Chou, A., Jaswinder, S.S. and Anthony, J.G. (2014) Frozen Section of the Pancreatic Neck Margin in Pancreatoduodenectomy for Pancreatic Adenocarcinoma Is of Limited Utility. Pathology, 46, 188-192. http://dx.doi.org/10.1097/PAT.0000000000000072 
[19] Menon, K.V., Gomez, D., Smith, A.M., Anthoney, A. and Verbeke, C.S. (2009) Impact of Margin Status on Survival Following Pancreatoduodenectomy for Cancer: The Leeds Pathology Protocol (LEEPP). HPB, 11, 18-24. http://dx.doi.org/10.1111/j.1477-2574.2008.00013.x

[20] Verbeke, C.S. and Menon, K.V. (2009) Redefining Resection Margin Status in Pancreatic Cancer. HPB, 11, $282-289$. http://dx.doi.org/10.1111/j.1477-2574.2009.00055.x

[21] Esposito, I., Kleeff, J., Bergmann, F., Reiser, C., Herpel, E., et al. (2008) Most Pancreatic Cancer Resections Are R1 Resections. Annals of Surgical Oncology, 15, 1651-1660. http://dx.doi.org/10.1245/s10434-008-9839-8

[22] Merkow, R.P., Bilimoria, K.Y., Bentrem, D.J., Pitt, H.A., Winchester, D.P., Posner, M.C., Ko, C.Y. and Pawlik, T.M. (2014) National Assessment of Margin Status as a Quality Indicator after Pancreatic Cancer Surgery. Annals of Surgical Oncology, 21, 1067-1074. http://dx.doi.org/10.1245/s10434-013-3338-2

[23] Landry, J., Catalano, P.J., Staley, C., Harris, W., Hoffman, J., et al. (2010) Randomized Phase II Study of Gemcitabine plus Radiotherapy versus Gemcitabine, 5-Fluorouracil, and Cisplatin Followed by Radiotherapy and 5-Fluorouracil for Patients with Locally Sdvanced, Potentially Resectable Pancreatic Adenocarcinoma. Journal of Surgical Oncology, 101, 587-592. http://dx.doi.org/10.1002/jso.21527

[24] Golcher, H., Brunner, T.B., Witzigmann, H., Marti, L., Bechstein, W., et al. (2015) Neoadjuvant Chemoradiation Therapy with Gemcitabine/Cisplatin and Surgery versus Immediate Surgery in Resectable Pancreatic Cancer: Results of the First Prospective Randomized Phase II Trial. Strahlentherapie und Onkologie, 191, 7-16. http://dx.doi.org/10.1007/s00066-014-0737-7

[25] Tachezy, M., Gebauer, F., Petersen, C., Arnold, D., Trepel, M., et al. (2014) Sequential Neoadjuvant Chemoradiotherapy (CRT) Followed by Curative Surgery vs. Primary Surgery Alone for Resectable, Non-Metastasized Pancreatic Adenocarcinoma: NEOPA-A Randomized Multicenter Phase III Study (NCT01900327, DRKS00003893, ISRCTN82191749). BMC Cancer, 14, 411. http://dx.doi.org/10.1186/1471-2407-14-411

[26] Andersen, J.R., Sørensen, S.M., Kruse, A., Rokkjaer, M. and Matzen, P. (1989) Randomised Trial of Endoscopic Endoprosthesis versus Operative Bypass in Malignant Obstructive Jaundice. Gut, 30, 1132-1135. http://dx.doi.org/10.1136/gut.30.8.1132

[27] Shepherd, H.A., Royle, G., Ross, A.P., Diba, A., Arthur, M., et al. (1988) Endoscopic Biliary Endoprosthesis in the Palliation of Malignant Obstruction of the Distal Common Bile Duct: A Randomized Trial. The British Journal of Surgery, 75, 1166-1168. http://dx.doi.org/10.1002/bjs.1800751207

[28] Seshadri, R.M., Ali, N., Warner, S., Cochran, A., Vrochides, D., et al. (2015) Training and Practice of the Next Generation HPB Surgeon: Analysis of the 2014 AHPBA Residents' and Fellows' Symposium Survey. HPB, 17, 1096-1104. http://dx.doi.org/10.1111/hpb.12498

[29] Lohse, I., Borgida, A., Cao, P., Cheung, M., Pintilie, M., et al. (2015) BRCA1 and BRCA2 Mutations Sensitize to Chemotherapy in Patient-Derived Pancreatic Cancer Xenografts. British Journal of Cancer, 113, 425-432. http://dx.doi.org/10.1038/bjc.2015.220

[30] Shao, T.F., Zheng, Y.T., Zhao, B., Li, T., Cheng, K.G. and Cai, W.M. (2014) Recombinant Expression of Different Mutant K-Ras Gene in Pancreatic Cancer Bxpc-3 Cells and Its Effects on Chemotherapy Sensitivity. Science China Life Sciences, 57, 1011-1017. http://dx.doi.org/10.1007/s11427-014-4724-0

[31] Narayanan, R. (2015) Phenome-Genome Association Studies of Pancreatic Cancer: New Targets for Therapy and Diagnosis. Cancer Genomics \& Proteomics, 12, 9-19. 